\title{
Research on highly sensitive optomagnetic sensor for rapid detection of inflammation
}

\author{
Chunxiu Liu, Haoyuan Cai, Jian Jia, Tianyang Cao, Chenghua Xu and Chang Liu* \\ State Key Laboratory of Transducer Technology, Institute of Electronics, Chinese Academy of Sciences, \\ Beijing, China
}

\begin{abstract}
.
BACKGROUND: C-reactive protein (CRP) is used to evaluate the evolution of infections and sepsis in critically ill patients. For POCT testing, biosensor-based detection techniques offer quick and convenient application.

OBJECTIVE: A prototype three dimensional chip was fabricated based on a new optomagnetic method to achieve the rapid detection of CRP.

METHODS: This work investigates a new technology for the quick quantitative detection of the C-reactive protein (CRP) by total internal reflection magnetic imaging (TIRMI) on a three dimensional optomagnetic sensor. Transparent glass and hydrophilic plastic film with channels were used to construct the three dimensional sensor. The magnetic nanoparticles and immunological reagent were immobilized on the reaction area of the sensor. Samples were detected using total internal reflection magnetic spot imaging (TIRMI) based on a sandwich magnetic immunoassay by one-step assay.

RESULTS: The developed 3D biosensor-TIRMI method showed a wide dynamic linear range $(0.2-200 \mathrm{ng} / \mathrm{ml})$ and quick detection (5 min) with low-sample volume $(10 \mu \mathrm{L})$.

CONCLUSIONS: We have presented a three dimensional optical protein chip that fulfills the demanding for point-of-care diagnostics in terms of ease-of-use (one step assay), miniaturization, assay time. This approach shows great promise for application in clinical investigations of biological samples.
\end{abstract}

Keywords: C-reactive protein, 3D optomagnetic sensor, total internal reflection magnetic imaging, all in one-step assay

\section{Introduction}

C-reactive protein (CRP) is an acute phase protein synthesized by the liver cells during inflammatory stimulation, such as microbial invasion, tissue damage and so on. Therefore, it is commonly used for differential diagnosis and monitoring. CRP is also used to evaluate the evolution of infections and sepsis in critically ill patients. The concentration of CRP is very low $(<5 \mathrm{mg} / \mathrm{L})$ in healthy human serum, can run up to $20-40 \mathrm{mg} / \mathrm{L}$ by viral infections, and significantly increase to more than $100 \mathrm{mg} / \mathrm{L}$ during bacterial infections. So it is considered to be valuable in disease condition judgment. A high level of plasma CRP is a risk factor as well as a marker for cardiovascular disease [1-6].

Currently, general detection of CRP requires large equipment and professional operators. For POCT testing, biosensor-based detection techniques offer quick and convenient application. The arraying technologies for sensor design have used a variety of substrates for microarray techniques to solve the 1 imitations and problems of the conventional detection method $[7,8]$.

\footnotetext{
${ }^{*}$ Corresponding author: Chang Liu, No.19, Beisihuan West Rd., Haidian District, Beijing, China. Tel.: +86 10 58887638; Fax: +86 10 58887638; E-mail: tuengineer@qq.com.
}

0928-7329/17/ $\$ 35.00$ (c) 2017 - IOS Press and the authors. All rights reserved

This article is published online with Open Access and distributed under the terms of the Creative Commons Attribution NonCommercial License (CC-BY-NC 4.0). 
In order to improve detection sensitivity and speed, the incorporation of magnetic particle enrichment into biosensor immunoassay procedures has been combined with the typical advantages associated with miniaturization. Magnetic micro-particles typically consist of a core of iron oxide nanoparticles and a polymer matrix. These particles have super-paramagnetic properties, because that the particles are only magnetic when a magnetic field is present. They quickly lose the magnetic properties and can re-disperse in a solution when the field removed.

The magnetic particles have very high surface-to-volume rations because of their small size. They are commercially available in a good range of surface functionalities. Therefore, they are useful for a variety of applications. Conventional magnetic particle-based assays, which involve the high efficiency, small volume, order transfer of reagents and washing buffers during the reaction, offer minimal particle loss and high capture efficiencies. However, they are batch processes, and their efficacy is reduced for having multiple operation steps that are both time-consuming and laborious. The combination of magnetic particles, sensor chips and total internal reflection imaging can reduce these multi-step procedures into a single step, decreasing processing times [9-16]. Magnetic imaging is a powerful tool for probing biological and physical systems. Previous studies have shown that TIR is a effective optical testing tool to investigate protein concentrations at a high signal to noise ratio [17-23]. The extremely low background noise (100-300 nm in wall-normal thickness) of the evanescent illumination generated by Total Internal Reflection has led to its widespread application [22]. Therefore, magnetic imaging and TIR may be combined for use in field detection [15,23].

This study demonstrates a method for the quick detection of the CRP by a single step using total internal reflection magnetic imaging (TIRMI) combined with arrayed 3D photo-magnetic biosensors. These aspects yield simple yet rapid detection by way of a bidirectional magnetron and three-dimensional magnetic speckle imaging on a sensor. This method cleverly uses magnetic particle enrichment, separation and magnetic imaging, avoiding the optical agent application and achieving one-step reaction. This method is applicable to a variety of detection fields.

\section{Materials and methods}

\subsection{Reagents}

Native CRP from human pleural fluid, monoclonal anti-human CRP antibody and monoclonal paired antibody functionalized with biotin to the human CRP were purchased from Abzymo Biosciences Co. (Beijing, China). Streptavidin Coated Magnetic Particles (500 nm diameter) were purchased from Ruihong Biosciences Co. (Shanghai, China). BSA and other reagents were purchased from Sigma-Aldrich Inc. (St. Louis, MO, USA). The buffer solutions were prepared with ultra-pure water ( $>18 \mathrm{M} \Omega$ ). Concentration values stated in this study are used by serial dilutions in PBS buffer.

\subsection{Apparatus and module}

The Nano-Plotter ${ }^{\mathrm{TM}}$ (GeSiM, Germany) device can be used to precisely and rapidly dispense pLto $\mu \mathrm{L}$-sized particles. The high precision laser engraving machine DPSS (Suzhou, China) was to cut biocompatible adhesive tape to form a microfluidic channel, a sample inlet, a waste liquid tank and a $\mu \mathrm{L}$ reaction chamber. For image and data collection, an optomagnetic analyzer consisting of TIRMI detection optics and an actuating electromagnetic module was created. 


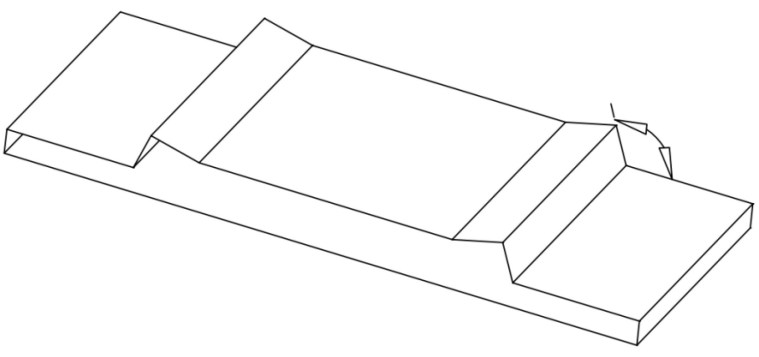

Fig. 1. 3D angle schematic diagram of the biosensor.

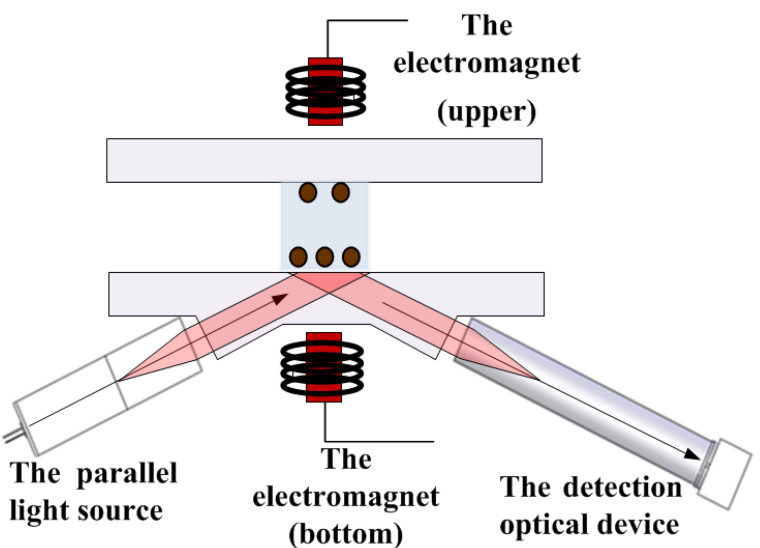

Fig. 2. Schematic diagram of the lab-made TIRMI system to test the CRP concentration by magnetic immunoassay with a 3D biosensor.

\subsection{Fabrication of sensor}

The sensors were fabricated by two layers. The glass devices had a sublayer with a 3-D optic angle (Fig. 1) and a smooth surface were precisely batch machined, and the cover high transparent plastic parts were laser cut. The microfluidic channel and the reaction chamber were formed between the two layers by laser-cut hydrophilic adhesive tape. The bottom surface of the reaction area was immobilized with monoclonal antibodies in order to serve as the sensing surface. Spots of the capture antibody were inkjet printed with a Nano-Plotter ${ }^{\mathrm{TM}}$ (GeSiM, Germany) using a $1 \mathrm{~nL}$ print volume. The top surface of the reaction chamber was modified by $5 \mu \mathrm{L}$ corresponding tracer antibody-magnetic composite particles.

\subsection{Laboratory-built TIRMI system}

Figure 2 shows the basic experimental setup of the TIRMI system containing the optics module and the magnetic module. A LED ( $380 \mathrm{~mW}$ at $625 \mathrm{~nm}$; Thorlabs, NJ, USA) was used as a light source. An MQ013MG-E2 CMOS (Ximea GmbH, Germany) camera combined telecentric lens was used to take magnetic spot images. The new electrically controlled bidirectional magnetic system is comprised of a power supply, an electromagnetic control circuit and program, and two identical small magnetic columns. The magnetic module can be adjusted to perform between 5 and 50 cycles of any time between 2 and 10 seconds. In this study, 20 cycles were performed at 5 seconds each.

\subsection{Detection method and data analysis method}

Ten $\mu \mathrm{L}$ of the standard sample was injected into the inlet of the sensor to perform the CRP detection tests. The sample passed through the channel into the reaction zone by means of capillary force and hydrophilic reaction. The tracer antibody-magnetic particles re-dispersed from the top surface and bound with the CRP in the reaction chamber. After complete filling and pre-reaction of the reaction zone, the bidirectional electric control magnetic actuation was started. The bidirectional magnetic force attracts the particles alternately up and down movement to increase the binding probability and speed of the immune reaction. A special magnetic washing step is used to remove excess magnetic particles after full immune response. The top electromagnet was turned on to draw unbound magnetic particles with the 


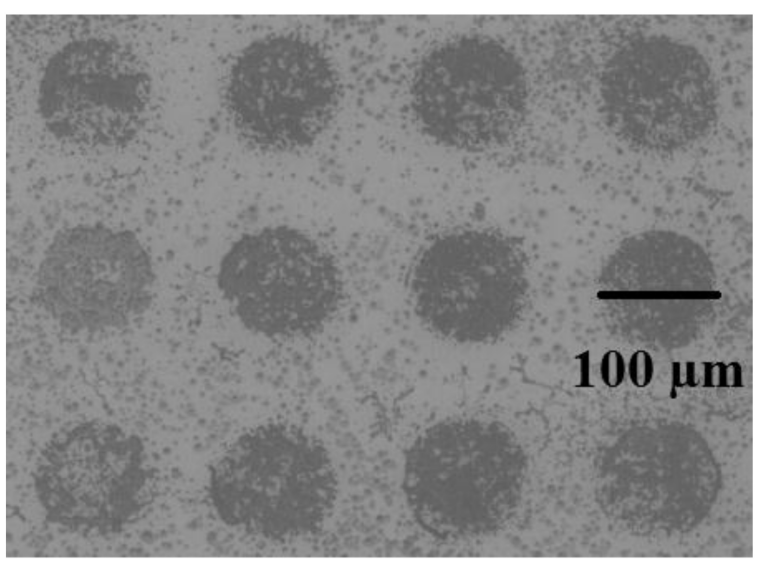

Fig. 3. TIRMI images of the array pots by standard CRP $(200 \mathrm{ng} / \mathrm{ml})$ on the biosensor by the magnetic immunoassay method.

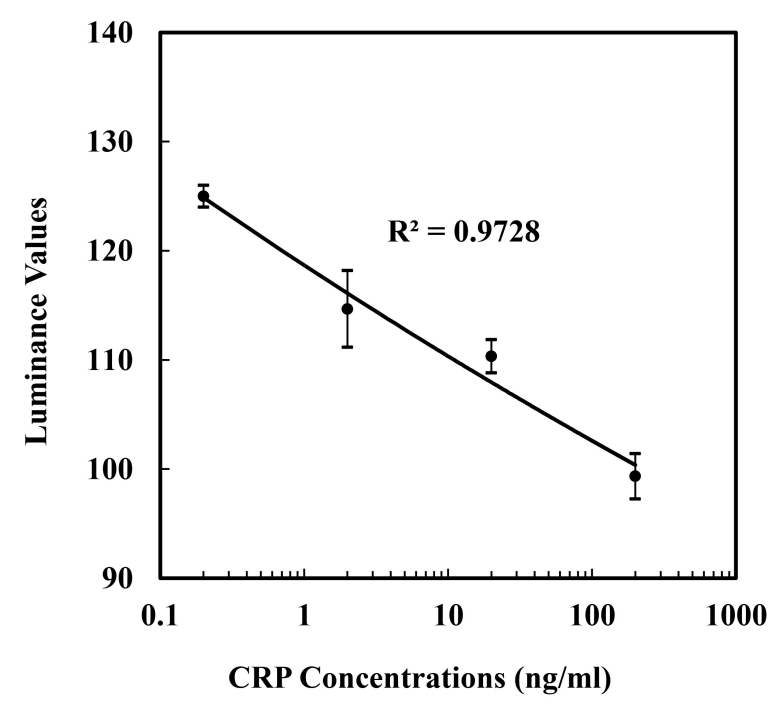

Fig. 4. Calibration curve of the negative correlation optical signal for the standard CRP. Data represent averages and standard deviations of 3 repetitions on different sensors.

bottom electromagnet turned off. The arrays formed by magnetic nanoparticles at the bottom surface of the sensor changed the TIR path and the changes of magnetic array spots were reflected by the optical method of TIRMI.

Matlab software was used to assay the obtained magnetic spot imaging and convert the images to data that are proportional to the concentration of CRP.

\section{Analysis and discussion}

This optomagnetic biosensor technology based on magnetic particles can be used for the detection of a broad range of biological molecules in quick assays. Figure 3 displays a TIRMI image of a assay for CRP with $200 \mathrm{ng} / \mathrm{ml}$. Nonspecific adhesion of magnetic particles to the surface outside the speckle region would interfere with imaging detection, which will be excluded in the following image analysis. Interference point elimination will be optimized in future surface modification and immunoassay research. Currently, using optics and electromagnets, 9 spots of $100 \mu \mathrm{m}$ diameter can be simultaneously imaged (Fig. 3). The number of spots imaged, and thus the number of detectable analytes, can be extended to 9 parameters.

Figure 4 shows the signal change of CRP concentrations ranging from 0.2 to $200 \mathrm{ng} / \mathrm{mL}$. The CRP samples were detected in a one-step method with immobilized reagents inside the micro reaction zone. The luminance values of the curve were derived from The TIRMI images of the arrays pots and assayed using a Matlab digital image processing program. The TIRMI grey values (negative correlation with luminance value) increased as the standard CRP concentration increased. The luminance values were negatively related to the logarithm of the CRP concentrations. The linear fit showed that the optical signal obtained is negative correlation with the CRP concentration during the range of $0.2 \sim 200 \mathrm{ng} / \mathrm{mL}$. This indicates that the sensor developed in this study can be useful for multi parameter detection. Further experimental analysis and software optimization work is being carried out to allow the detection of more parameters. 


\section{Conclusion}

The quick assay of standard CRP was researched in order to develop applicable 3D devices and optomagnetic methods for precise and fast disease diagnosis at low concentrations with small sample of microlitre level. Compared to the traditional detection technique, the developed 3D biosensor-TIRMI method showed a wide dynamic linear range $(0.2-200 \mathrm{ng} / \mathrm{ml})$ and quick detection $(5 \mathrm{~min})$ with lowsample volume $(10 \mu \mathrm{L})$. Therefore, this approach shows great promise for application in clinical investigations of biological samples with low concentrations. In addition, the clear advantages of the presence of 3D devices and optomagnetic methods are likely extensible to other targets for quick testing.

\section{Acknowledgments}

This work is supported by the NSFC (No.60801032, 61271147, 61372052), the major national scientific research plan (2011CB933202), the project of CAS (KFJ-EW-STS-046) and the high resolution major projects (06-Y30B04-9002-13/15).

\section{Conflict of interest}

None to report.

\section{References}

[1] Meisner M, Adina H and Schmidt J, Correlation of procalcitonin and C-reactive protein to inflammation, complications, and outcome during the intensive care unit course of multiple-trauma patients, Critical Care, 2005, 10(1): 1-10.

[2] Sun HJ, Tomonari K, Tomonaga I, Kinta H, Masashi S, Zhang B, Shuji K, Masatoshi M, Teruo W, Yujiro A, Chen E and Fan JL, C-Reactive Protein in Atherosclerotic Lesions-Its Origin and Pathophysiological Significance, American Journal of Pathology, 2005, 167(4): 1139-1148.

[3] Gian PC, Claudio P, Michael M, Antonio S, Daniela B and Laura S, Procalcitonin and C-reactive protein during systemic inflammatory response syndrome, sepsis and organ dysfunction, Critical Care, 2004, 8(4): R234-R242.

[4] Gavela T, Cabeza B and Serrano A, C-reactive protein and procalcitonin are predictors of the severity of acute appendicitis in children, Pediatr Emerg Care, 2012, 28(5): 416-419.

[5] Michael M, Klaus T, Thomas P and Joachim S, Comparison of procalcitonin (PCT) and C-reactive protein (CRP) plasma concentrations at different SOFA scores during the course of sepsis and MODS, Crit Care, 1999, 3: 45-50.

[6] Mark BP and Gideon MH, C-reactive protein: a critical update, J. Clin. Invest, 2003, 111: 1805-1812.

[7] Patricia B, Ursula S, Hedvig TS and Claudia P, Miniaturized protein microarray with internal calibration as point-of-care device for diagnosis of neonatal sepsis, Sensors, 2012, 12: 1494-1508.

[8] Daniel M, Stefan H, Gunter R, Felix VS and Roland Z, Microfluidic lab-on-a-chip platforms: requirements, characteristics and applications, Chem. Soc. Rev., 2010, 39: 1153-1182.

[9] Lacharme F, Vandevyver C and Gijs MAM, Magnetic beads retention device for sandwich immunoassay: comparison of off-chip and on-chip antibody incubation, Microfluid Nanofluid, 2009, 7: 479-487.

[10] Czilwik MJG, Auwaerter V, Roth G, Zengerle R and Stetten F, Continuous microfluidic DNA extraction using phasetransfer magnetophoresis, Lab Chip, 2010, 10: 3284-3290.

[11] Hoshino K, Huang YY, Lane N, Huebschman M, Uhr JW, Frenkel EP and Zhang X, Microchip-based immunomagnetic detection of circulating tumor cells, Lab Chip, 2011, 11: 3449-3457.

[12] Chayakom P, Ender Y, Mark DT, Sebastiaan JT, Thomas H, Nicole P and Paul V, Phaseguide assisted liquid lamination for magnetic particle-based assays, Lab Chip, 2014, 14: 2334-2343.

[13] Alexander R, Arthur MJ, Jaap MJT and Menno WJP, Integrated lab-on-chip biosensing systems based on magnetic particle actuation - a comprehensive review, Lab Chip, 2014, 14: 1966-1986.

[14] Nicole P, On-chip bioanalysis with magnetic particles, Current Opinion in Chemical Biology, 2012, 16(3-4): $436-443$. 
[15] Islam MS, Lee HG, Choo J, Song JM and Kang SH, High sensitive detection of C-reactive protein by total internal reflection fluorescence microscopy on rapidly making nanoarray protein chip, Talanta, 2010, 81(4-5): 1402-1408.

[16] Srinivasan R, Daniel AC, Arjan PQ and Ratnesh L, High performance, LED powered, waveguide based total internal reflection microscopy, Scientific Reports, 2013, 3: 2133.

[17] Nam CHL, Dzung VD, Ryuji Y, John W and Susumu S, Dual-color Total Internal Reflection (TIR)-based Chip for Simultaneous Detection of Two Fluorophores, IEEE Sensors Conference, 2008, 1191-1194.

[18] Vigneshwaran M, Bhaskara VC and James FR, Magnetic particles in ultrasensitive biomarker protein measurements for cancer detection and monitoring, Expert Opin Med Diagn, 2011, 5(5): 381-391.

[19] Rémy F, Aurélien B, Christophe E, He QH, Samuel C, Ali B and Anne MG, On chip magnetic actuator for batch-mode dynamic manipulation of magnetic particles in compact lab-on-chip, Sensors and Actuators B, 2011, 160: 1520-1528.

[20] Martin AMG, Applications of magnetic labs-on-a-chip, Microfluidics Based Microsystems: Fundamentals and Applications, Springer Science Business Media B.V., 2010, 453-465.

[21] Martin AMG, Magnetic bead handling on-chip: new opportunities for analytical applications, Microfluidics and Nanofluidics, 2004, 1: 22-40.

[22] Nam CHL, Ryuji Y, Dzung VD, Thien DN, John CW and Susumu S, Versatile microfluidic total internal reflection (TIR)-based devices: Application to microbeads velocity measurement and single molecule detection with upright and inverted microscope, Lab Chip, 2009, 9: 244-250.

[23] Sage DL, Arai K, Glenn DR, DeVience SJ, Pham LM, Lukin MD, Yacoby A, Komeili A and Walsworth RL, Optical magnetic imaging of living cells, Nature, 2013, 496: 486-489. 